NSF-ITP-93-46

CALT-68-1863

TIFR-TH-93-19

hep-th/9304154

April 1993

\title{
DUALITY SYMMETRIC ACTIONS
}

\author{
JOHN H. SCHWARz ${ }^{\star \dagger}$ \\ Institute for Theoretical Physics, University \\ of California, Santa Barbara, CA 93106 \\ California Insitute of Technology, Pasadena, CA 91125 \\ and \\ Ashoke Sen $§ \uparrow$ \\ Institute for Theoretical Physics, University \\ of California, Santa Barbara, CA 93106 \\ Tata Institute of Fundamental Research, Homi \\ Bhabha Road, Bombay 400005, India
}

* Supported in part by the U.S. Dept. of Energy under Grant No. DE-FG03-92-ER40701.

$\dagger$ JHS@THEORY3.CALTECH.EDU, JHS@NSFITP.ITP.UCSB.EDU

$\ddagger$ Permanent address.

$\S$ SEN@TIFRVAX.BITNET, SEN@SBITP.UCSB.EDU

I Supported in part by National Science Fundation under grant No. PHY89-04035. 


\begin{abstract}
It is frequently useful to construct dual descriptions of theories containing antisymmetric tensor fields by introducing a new potential whose curl gives the dual field strength, thereby interchanging field equations with Bianchi identities. We describe a general procedure for constructing actions containing both potentials at the same time, such that the dual relationship of the field strengths arises as an equation of motion. The price for doing this is the sacrifice of manifest Lorentz invariance or general coordinate invariance, though both symmetries can be realized nonetheless. There are various examples of global symmetries that have been realized as symmetries of field equations but not actions. These can be elevated to symmetries of the action by our method. The main example that we focus on is the low-energy effective action description of the heterotic string theory compactified on a six-torus to four dimensions. We show that the SL(2,R) symmetry, whose SL(2,Z) subgroup has been conjectured to be an exact symmetry of the full string theory, can be realized on the action in a way that brings out a remarkable similarity to the target space duality symmetry $\mathrm{O}(6,22)$. Our analysis indicates that $\mathrm{SL}(2, \mathrm{Z})$ symmetry may arise naturally in a dual formulation of the theory.
\end{abstract}




\section{Introduction}

Montonen and Olive [1] conjectured in 1977 that some theories with a spontaneously broken gauge symmetry possess a duality symmetry that interchanges electrically charged elementary particles with magnetically charged t' Hooft-Polyakov monopoles. Such a symmetry would relate strong coupling to weak coupling, since it sends the coupling constant to its inverse. Later analysis showed that among fourdimensional field theories the best candidate for realizing the Montonen-Olive duality conjecture is the globally supersymmetric $N=4$ Yang-Mills-Higgs system [2]. A similar duality conjecture for ten dimensions that would relate strong coupling in string theory to weak coupling in five-brane theory was made in refs. [3-6] .

Apparently unrelated work, at about the same time as the Montonen-Olive conjecture, showed that many extended supergravity theories in four dimensions have global non-compact symmetries [7] [8]. Some of these symmetries were realized as symmetries of the action, whereas others were only demonstrated to be symmetries of the equations of motion. In particular, many of these theories contain an $\mathrm{SU}(1,1)$ symmetry (or, equivalently, an $\mathrm{SL}(2, \mathrm{R})$ symmetry), which is a symmetry of the equations of motion only.

Ref.[9] investigated dimensional reduction of the bosonic sector of $N=1$ supergravity theory in ten dimensions, coupled to a set of abelian gauge field supermultiplets, to four dimensions. The resulting action describes the bosonic part of the low-energy effective field theory for the heterotic string theory compactified on a six-dimensional torus at a generic point in the moduli space, where all non-abelian symmetries are broken. The action of this theory has a manifest global $\mathrm{O}(6,22)$ symmetry. A discrete $\mathrm{O}(6,22 ; \mathrm{Z})$ subgroup of this, which is a symmetry of the Narain lattice [10] [11], can be shown to be an exact symmetry of the compactified string theory at each order of the string loop perturbation expansion.

This dimensionally reduced theory also turns out to have a hidden $\mathrm{SL}(2, \mathrm{R})$ symmetry,[12 - 14] which is only a symmetry of the equations of motion and not of the action. Part of this symmetry is broken by the instanton corrections in string 
theory. It was conjectured in refs.[13][14] that the remaining subgroup, which turns out to be the discrete group $\mathrm{SL}(2, \mathrm{Z})$, may be an exact symmetry of the heterotic string theory compactified on a six-dimensional torus. (This suggestion was originally made in the context of a generic four-dimensional string theory by Ibáñez et. al.[15] based on the analysis of the scalar sector of these theories.) It was argued in ref.[13] that since the elementary strings can be regarded as soliton solutions in this effective field theory [16] [17], SL(2,Z) invariance of the effective field theory may be all that is required to establish $\mathrm{SL}(2, \mathrm{Z})$ invariance of the full string theory. Further support for SL(2,Z) symmetry in toroidally compactified heterotic string theory was provided by noting that the spectrum of electric and magnetic charges, and also the known part of the mass spectrum of the supersymmetric states in this theory, are all consistent with the proposed SL(2,Z) symmetry [18] [19].

Although $\mathrm{SL}(2, \mathrm{R}) \times \mathrm{O}(6,22)$ appears as a symmetry of the classical equations of motion of the low-energy effective action, the two factors seem to be on a somewhat different footing: $\mathrm{O}(6,22)$ is a symmetry of the effective action, whereas $\mathrm{SL}(2, \mathrm{R})$ is only a symmetry of the equations of motion. Also, the discrete subgroup $\mathrm{O}(6,22 ; \mathrm{Z})$ is a symmetry of the string spectrum at the string tree level, but the discrete subgroup $\mathrm{SL}(2, \mathrm{Z})$ is certainly not a symmetry of tree level string theory (though it could be a symmetry of the full non-perturbative string theory), since it interchanges string states with 't Hooft-Polyakov-type monopole solutions.

One of the main purposes of this paper is to reformulate the theory in such a way that, at least in the context of low-energy effective field theory, the $\mathrm{O}(6,22)$ and $\mathrm{SL}(2, \mathrm{R})$ symmetries appear on a more or less equal footing. In particular, we shall rewrite the dimensionally reduced action in such a way that both the $\mathrm{O}(6,22)$ and the $\mathrm{SL}(2, \mathrm{R})$ transformations appear as symmetries of the action, and not just of the equations of motion. The price that must be paid for this is manifest general coordinate invariance of the action, though the action does have general coordinate invariance. The way this works is that the action is invariant under a symmetry that reduces to the usual general coordinate transformations when certain auxiliary fields are eliminated by their equations of motion. Actually, spatial 
reparametrization invariance remains manifest.

We start in sect.2 with a very simple system that illustrates the key new feature of our construction, namely, free Maxwell theory. In the usual formulation, the equations of motion of Maxwell theory (without sources) are symmetric under the duality transformation $\vec{E} \rightarrow \vec{B}, \vec{B} \rightarrow-\vec{E}$, but the action is not. We show that by introducing appropriate auxiliary fields it is possible to make this duality a manifest symmetry of the action. Although this process sacrifices manifest Lorentz invariance, the action is invariant under a certain set of transformations that reduce to the usual Lorentz transformations when the auxiliary fields are eliminated by their equations of motion. We show how to couple this theory to gravity and to make it supersymmetric while maintaining manifest duality symmetry. Generalizations to higher dimensions and other systems are discussed briefly.

In sect. 3 the formalism developed in sect. 2 is used to write down an action that is equivalent to the action of the dimensionally reduced $D=10 N=1$ supergravity theory, but which has manifest $\mathrm{O}(6,22)$ and $\mathrm{SL}(2, \mathrm{R})$ invariance. In this form of the action, the fields that transform under $\mathrm{SL}(2, \mathrm{R})$ and $\mathrm{O}(6,22)$ are treated quite symmetrically. In this sense, $\mathrm{SL}(2, \mathrm{R})$ and $\mathrm{O}(6,22)$ appear to be on equal footing. This action is not manifestly general coordinate invariant, but (as above) it does have general coordinate invariance nevertheless.

When the auxiliary fields of the $\mathrm{SL}(2, \mathrm{R}) \times \mathrm{O}(6,22)$ invariant action are eliminated by their equations of motion, the original action of ref.[9] is recovered. In the special case where the various four-dimensional fields that originate as components of $\mathrm{U}(1)^{16}$ gauge fields in ten dimensions are set to zero, there is no preferred choice as to which fields should be regarded as auxiliary. In particular, choosing a different set of fields in the manifestly $\mathrm{SL}(2, \mathrm{R}) \times \mathrm{O}(6,22)$ invariant formulation to be the auxiliary fields, and eliminating them by their equations of motion, gives rise to a manifestly $\mathrm{SL}(2, \mathrm{R})$ and general coordinate invariant formulation of the theory (at the sacrifice of manifest $\mathrm{O}(6,22)$ symmetry).

Although this analysis puts $\mathrm{SL}(2, \mathrm{R})$ and $\mathrm{O}(6,22)$ symmetry on a very symmet- 
ric footing from the point of view of the four-dimensional effective field theory, the $\mathrm{O}(6,22)$ invariant formulation of the theory could be regarded as more fundamental, since it is the formulation that appears naturally in the dimensional reduction of the $N=1$ supergravity theory from ten to four dimensions. However, in sect.4 we remove this asymmetry by showing that it is the $\mathrm{SL}(2, \mathrm{R})$ and general coordinate invariant (but not manifestly $\mathrm{O}(6,22)$ invariant) formulation that arises naturally in the dimensional reduction of the dual formulation of the $N=1$ supergravity theory in ten dimensions based on a six-form potential with a seven-form field strength. Since the fields in this dual formulation couple more naturally to the five-brane [4], we speculate that the $\mathrm{SL}(2, \mathrm{Z})$ symmetry may have a more natural realization in the theory of five-branes. In particular, we show that when expressed in terms of the natural variables of the five-brane theory, the complex field that transforms under the $\mathrm{SL}(2, \mathrm{Z})$ symmetry takes a form very similar to the fields that transformed under the target space duality symmetry, expressed in terms of the natural variables of the string theory.

Sect. 5 gives a summary of our results and some comments. In particular, we comment on a possible reformulation of the $N=1$ supergravity action in ten dimensions, which, upon dimensional reduction, would give the manifestly $\operatorname{SL}(2, \mathrm{R})$ invariant form of the effective action even when the $\mathrm{U}(1)^{16}$ gauge fields in ten dimensions are included in the theory.

The appendix contains part of the analysis involved in the dimensional reduction of the dual formulation of $D=10 N=1$ supergravity theory. 


\section{Duality Invariant Einstein-Maxwell Action}

In this section we discuss the construction of an action that is equivalent to the usual Einstein-Maxwell action, but is manifestly invariant under a duality symmetry that reduces to the usual $\vec{E} \rightarrow \vec{B}, \vec{B} \rightarrow-\vec{E}$ symmetry when the auxiliary fields of the theory are eliminated by their equations of motion. The method that we use is very similar to one introduced by Henneaux and Teitelboim to solve the problem of constructing an action for the self-dual $(2 q+1)$-form field strength in $4 q+2$ dimensions [20]. In the special case of two dimensions, it was discovered independently by Floreanini and Jackiw [21] and used by Tseytlin for the construction of a manifestly duality invariant scalar field theory in two dimensions [22]. In each of these papers, the key ingredient was to give up manifest Lorentz invariance of the action. This will also be the key ingredient in our construction. One of the main differences between the analysis of the papers mentioned above and our analysis is the dimensionality of space-time; whereas the analysis of the previous papers are applicable in $2,6,10, \ldots$ dimansions, our analysis will be in 4 dimensions. However, at the end of this section we shall discuss the generalization of our analysis to any dimension. We also clarify the relationship between our results and those of ref.[20].

This section will be divided into five subsections. In subsection 2.1, we present an action in four dimensions, which has manifest duality symmetry and is equivalent to free Maxwell theory. The action reduces to Maxwell's action when the auxiliary fields are eliminated by their equations of motion. Although this action is not manifestly Lorentz invariant, we shall show that the action is, in fact, invariant under a set of transformations that reduce to the standard Lorentz transformations when the auxiliary fields are eliminated by their equations of motion. In subsection 2.2 we show how to couple this theory to gravity while preserving manifest duality symmetry. This gives rise to a theory that is not manifestly invariant under general coordinate transformations, but is invariant under a set of transformations that reduce to the usual general coordinate transformations when 
the auxiliary fields are eliminated by their equations of motion. Furthermore, the action reduces to the usual Maxwell action in curved space-time when the auxiliary fields are eliminated by their equations of motion. In subsection 2.3 the construction is generalized to a field theory of $p$-form fields in $2 p+2$ dimensions for any integer $p$, and the relationship between our action and that of ref.[20] is discussed. In subsection 2.4, the construction of subsection 2.1 is generalized to the field theory of $m$-form fields in $d$ dimensions for any $m$ and $d$, and the action is written in a form in which the original field, and the dual $(d-m-2)$-form field appear on an equivalent footing. Finally, in subsection 2.5 we show how to supersymmetrize our version of Maxwell's action (as described in subsection 2.1), while preserving manifest duality symmetry.

\subsection{Duality Invariant ACtion}

The basic idea of our construction is to introduce independent gauge fields for the electromagnetic field strength and its dual. The fact that the two field strengths are the duals of one another is then arranged to be a consequence of the equations of motion. Accordingly, the basic field variables of our action are a pair

of gauge fields $A_{\mu}^{(\alpha)}(0 \leq \mu \leq 3,1 \leq \alpha \leq 2)$. We begin with flat space-time. The appropriate action is then

$$
S=-\frac{1}{2} \int d^{4} x\left(B^{(\alpha) i} \mathcal{L}_{\alpha \beta} E_{i}^{(\beta)}+B^{(\alpha) i} B^{(\alpha) i}\right)
$$

where

$$
E_{i}^{(\alpha)}=\partial_{0} A_{i}^{(\alpha)}-\partial_{i} A_{0}^{(\alpha)}, \quad B^{(\alpha) i}=\epsilon^{i j k} \partial_{j} A_{k}^{(\alpha)} \quad 1 \leq i, j, k \leq 3
$$

and

$$
\mathcal{L}=\left(\begin{array}{cc}
0 & 1 \\
-1 & 0
\end{array}\right)
$$

This action has the following gauge invariances

$$
\delta A_{0}^{(\alpha)}=\Psi^{(\alpha)}, \quad \delta A_{i}^{(\alpha)}=\partial_{i} \Lambda^{(\alpha)} .
$$


Using the gauge transformation parameter $\Psi^{(\alpha)}$, we can set

$$
A_{0}^{(\alpha)}=0
$$

Since $A_{0}^{(\alpha)}$ only appears as part of a total derivative in the action, no equations of motion are lost. (This is to be contrasted with choosing $A_{0}=0$ gauge in the usual formulation of Maxwell theory.) The equation of motion of the field $A_{i}^{(2)}$ now gives

$$
\epsilon^{i j k} \partial_{j}\left(B^{(2) k}-E_{k}^{(1)}\right)=0 .
$$

Since this does not involve any time derivative of $A_{i}^{(2)}$, we can treat $A_{i}^{(2)}$ as an auxiliary field, and eliminate it from the action (2.1) by using eq.(2.6). Eq.(2.6) gives

$$
B^{(2) k}=E_{k}^{(1)}+\partial_{k} \phi
$$

for some $\phi$. Using the freedom associated with the gauge transformation parameter $\Lambda^{(1)}$, we can set $\phi=0$, so that eq.(2.7) reduces to

$$
B^{(2) k}=E_{k}^{(1)}
$$

Substituting the value of $B^{(2) k}$ given in eq.(2.8) into the action (2.1), we get back the usual Maxwell action for the field $A_{\mu}^{(1)}$

$$
-\frac{1}{2} \int d^{4} x\left(B^{(1) i} B^{(1) i}-E_{i}^{(1)} E_{i}^{(1)}\right)
$$

in the gauge $A_{0}^{(1)}=0$. The Gauss's law constraint, $\partial_{i} E_{i}^{(1)}=0$, is a consequence of the Bianchi identity for $B^{(2) k}$ in eq. (2.8). Note that (2.1) is first order in time derivatives, and therefore it is well-suited to a Hamiltonian analysis. 
We now return to the original action $S$ given in eq.(2.1) and study its symmetries. First of all we note that this action is manifestly invariant under the duality symmetry

$$
A_{\mu}^{(\alpha)} \rightarrow \mathcal{L}_{\alpha \beta} A_{\mu}^{(\beta)}
$$

which implies the transformation

$$
\left(\begin{array}{c}
B^{(1) i} \\
E_{i}^{(1)}
\end{array}\right) \rightarrow \mathcal{L}\left(\begin{array}{c}
B^{(1) i} \\
E_{i}^{(1)}
\end{array}\right)
$$

when we use the equation of motion (2.8) of $A_{i}^{(2)}$. Note that in the usual formulation of Maxwell's theory, the duality transformation is a highly non-local transformation on the vector potential. In contrast, here it is a local transformation on the fields $A_{\mu}^{(\alpha)}$.

The action given in eq.(2.1) is manifestly invariant under rotations, but not manifestly invariant under Lorentz boosts. Nevertheless, it can be checked easily that the action is invariant under the following transformation in the $A_{0}^{(\alpha)}=0$ gauge:

$$
\delta A_{i}^{(\alpha)}=x^{0} v^{k} \partial_{k} A_{i}^{(\alpha)}+\vec{v} \cdot \vec{x} \mathcal{L}_{\alpha \beta} \epsilon^{i j k} \partial_{j} A_{k}^{(\beta)}
$$

where $\vec{v}$ is an arbitrary constant three-dimensional vector. Furthermore, if we use the equations of motion (2.8), the above transformation reduces to

$$
\delta A_{i}^{(1)}=x^{0} v^{k} \partial_{k} A_{i}^{(1)}+\vec{v} \cdot \vec{x} \partial_{0} A_{i}^{(1)}
$$

which is the usual Lorentz transformation law of the field $A_{i}^{(1)}$ in the $A_{0}^{(1)}=0$ gauge. 


\subsection{Coupling to Gravity}

We shall now generalize the action (2.1) to curved space-time in such a way that when the fields $A_{\mu}^{(2)}$ are eliminated using their equations of motion, we recover the Maxwell action for the field $A_{\mu}^{(1)}$ in curved space-time

$$
-\frac{1}{4} \int d^{4} x \sqrt{-g} g^{\mu \rho} g^{\nu \sigma} F_{\mu \nu}^{(1)} F_{\rho \sigma}^{(1)}
$$

In order to do this, we start with the most general form of the action that is first order in time derivatives, invariant under the duality transformation (2.10), and invariant under the gauge transformations (2.4). This is given by

$$
S_{g}=-\frac{1}{2} \int d^{4} x\left[B^{(\alpha) i} \mathcal{L}_{\alpha \beta} E_{i}^{(\beta)}+t_{i j} B^{(\alpha) i} B^{(\alpha) j}+u_{i j} B^{(\alpha) i} \mathcal{L}_{\alpha \beta} B^{(\beta) j}\right] .
$$

Here $t_{i j}$ and $u_{i j}$ are unknown coefficients that are determined by first eliminating the fields $A_{i}^{(2)}$ from the action (2.15) by using their equations of motion, and then demanding that the resulting action is identical to the action (2.14). It turns out that this procedure determines the coefficients $t_{i j}$ and $u_{i j}$ uniquely. The final action obtained this way is given by

$$
S_{g}=-\frac{1}{2} \int d^{4} x\left[B^{(\alpha) i} \mathcal{L}_{\alpha \beta} E_{i}^{(\beta)}-\frac{g_{i j}}{\sqrt{-g} g^{00}} B^{(\alpha) i} B^{(\alpha) j}+\epsilon^{i j k} \frac{g^{0 k}}{g^{00}} B^{(\alpha) i} \mathcal{L}_{\alpha \beta} B^{(\beta) j}\right] .
$$

Here, as in eq. (2.14), $\sqrt{-g}=\sqrt{-\operatorname{det}\left(g_{\mu \nu}\right)}$ and $g^{\mu \nu}$ is the inverse of $g_{\mu \nu}$, the ordinary four-dimensional metric. These conventions are retained even when space and time components are enumerated separately. By rewriting this formula in terms of $F_{i j}^{(\alpha)}$ instead of $B^{(\alpha) k}$ general coordinate invariance in the spatial directions becomes manifest.

The action $S_{g}$ is manifestly invariant under the duality transformation (2.10) and the gauge transformations (2.4). Although $S_{g}$ is not manifestly invariant 
under general coordinate transformations, it can be shown to be invariant under the following transformation:

$$
\delta A_{i}^{(\alpha)}=\xi^{j} \partial_{j} A_{i}^{(\alpha)}+\left(\partial_{i} \xi^{j}\right) A_{j}^{(\alpha)}+\xi^{0}\left\{-\frac{g_{i j}}{\sqrt{-g} g^{00}} \mathcal{L}_{\alpha \beta} B^{(\beta) j}-\frac{g^{0 k}}{g^{00}} \epsilon^{i j k} B^{(\alpha) j}\right\} .
$$

To see the connection between this transformation and the usual general coordinate transformation, we eliminate $A_{i}^{(2)}$ using its equation of motion. In the $A_{0}^{(\alpha)}=0$ gauge, the $A_{i}^{(2)}$ equation of motion is given by

$$
\epsilon^{i j k} \partial_{j}\left\{E_{k}^{(1)}+\frac{g_{k j}}{\sqrt{-g} g^{00}} B^{(2) j}+\epsilon^{k l m} \frac{g^{0 m}}{g^{00}} B^{(1) l}\right\}=0
$$

Choosing the gauge transformation parameter $\Lambda^{(1)}$ appropriately, this equation can be integrated to the form

$$
E_{k}^{(1)}+\frac{g_{k j}}{\sqrt{-g} g^{00}} B^{(2) j}+\epsilon^{k l m} \frac{g^{0 m}}{g^{00}} B^{(1) l}=0
$$

If we now substitute the expression for $A_{i}^{(2)}$ obtained from eq.(2.19) into the expression for $\delta A_{i}^{(1)}$ given in eq.(2.17), we get

$$
\delta A_{i}^{(1)}=\xi^{j} \partial_{j} A_{i}^{(1)}+\left(\partial_{i} \xi^{j}\right) A_{j}^{(1)}+\xi^{0} \partial_{0} A_{i}^{(1)}
$$

This is the standard general coordinate transformation law of a vector under an infinitesimal coordinate transformation $x^{\mu} \rightarrow x^{\mu}+\xi^{\mu}$ in the $A_{0}^{(1)}=0$ gauge.

\subsection{Generalization to $p$-Form Fields in $2 p+2$ Dimensions}

In $2 p+2$ dimensions, we start with a pair of $p$-form gauge potentials $A_{\mu_{1} \ldots \mu_{p}}^{(\alpha)}$ 
$\left(0 \leq \mu_{k} \leq 2 p+1,1 \leq \alpha \leq 2\right)$, and define

$$
\begin{aligned}
E_{i_{1} \ldots i_{p}}^{(\alpha)} & =\partial_{0} A_{i_{1} \ldots i_{p}}^{(\alpha)}-(-1)^{p} \partial_{\left[i_{1}\right.} A_{\left.i_{2} \ldots i_{p}\right] 0}^{(\alpha)}, \\
B^{(\alpha) i_{1} \ldots i_{p}} & =\frac{1}{p !} \epsilon^{i_{1} \ldots i_{p} j_{1} \ldots j_{p+1}} \partial_{j_{1}} A_{j_{2} \ldots j_{p+1}}^{(\alpha)},
\end{aligned}
$$

and

$$
\mathcal{L}^{(p)}=\left(\begin{array}{cc}
0 & 1 \\
(-1)^{p} & 0
\end{array}\right)
$$

In terms of these quantities, the generalization of the action (2.1) is given by

$$
S=-\frac{1}{2 . p !} \int d^{2 p+2} x\left[B^{(\alpha) i_{1} \ldots i_{p}} \mathcal{L}_{\alpha \beta}^{(p)} E_{i_{1} \ldots i_{p}}^{(\beta)}+B^{(\alpha) i_{1} \ldots i_{p}} B^{(\alpha) i_{1} \ldots i_{p}}\right] .
$$

This action is invariant under the gauge transformations

$$
\delta A_{0 i_{1} \ldots i_{p-1}}^{(\alpha)}=\Psi_{i_{1} \ldots i_{p-1}}^{(\alpha)}, \quad \delta A_{i_{1} \ldots i_{p}}^{(\alpha)}=\partial_{\left[i_{1}\right.} \Lambda_{\left.i_{2} \ldots i_{p}\right]}^{(\alpha)}
$$

the duality transformation

$$
A_{\mu_{1} \ldots \mu_{p}}^{(\alpha)} \rightarrow \mathcal{L}_{\alpha \beta}^{(p)} A_{\mu_{1} \ldots \mu_{p}}^{(\beta)}
$$

and the 'Lorentz transformation'

$$
\delta A_{i_{1} \ldots i_{p}}^{(\alpha)}=x^{0} v^{j} \partial_{j} A_{i_{1} \ldots i_{p}}^{(\alpha)}+(-1)^{p+1} \vec{v} \cdot \vec{x} \mathcal{L}_{\alpha \beta}^{(p)} B^{(\beta) i_{1} \ldots i_{p}} .
$$

Using the gauge transformation parameter $\Psi^{(\alpha)}$ we can set the gauge $A_{0 i_{1} \ldots i_{p-1}}^{(\alpha)}=0$. If we now eliminate the fields $A_{i_{1} \ldots i_{p}}^{(2)}$ using their equations of motion, we recover the standard action for a $(p+1)$-form field strength in $2 p+2$ dimensions

$$
-\frac{1}{2 .(p+1) !} \int d^{2 p+2} x F_{\mu_{1} \ldots \mu_{p+1}}^{(1)} F_{\nu_{1} \ldots \nu_{p+1}}^{(1)} \eta^{\mu_{1} \nu_{1}} \ldots \eta^{\mu_{p+1} \nu_{p+1}}
$$

where

$$
F_{\mu_{1} \ldots \mu_{p+1}}^{(1)}=\partial_{\left[\mu_{1}\right.} A_{\left.\mu_{2} \ldots \mu_{p+1}\right]}^{(1)}
$$

Also, in this case the Lorentz transformation law of $A_{i_{1} \ldots i_{p}}^{(1)}$ takes the standard form in the $A_{0 i_{1} \ldots i_{p-1}}^{(1)}=0$ gauge. 
For $p$ even, the matrix $\mathcal{L}_{\alpha \beta}^{(p)}$ can be diagonalized to the form $\operatorname{Diag}(1,-1)$. The action (2.23) then describes the direct sum of two decoupled theories. One of them is described by the action of a self-dual $(p+1)$-form field strength as written down in ref.[20], the other is described by the action of an anti-self-dual $(p+1)$-form field strength.

Coupling this theory to gravity involves a straightforward generalization of eq.(2.16). The corresponding action is given by

$$
\begin{aligned}
S_{g}= & -\frac{1}{2 \cdot p !} \int d^{2 p+2} x\left[B^{(\alpha) i_{1} \ldots i_{p}} \mathcal{L}_{\alpha \beta}^{(p)} E_{i_{1} \ldots i_{p}}^{(\beta)}-\frac{g_{i_{1} j_{1} \ldots g} \ldots g_{i_{p} j_{p}}}{\sqrt{-g} g^{00}} B^{(\alpha) i_{1} \ldots i_{p}} B^{(\alpha) j_{1} \ldots j_{p}}\right. \\
& \left.+\frac{1}{p !} \epsilon^{i_{1} \ldots i_{p} j_{1} \ldots j_{p} k} \frac{g^{0 k}}{g^{00}} B^{(\alpha) i_{1} \ldots i_{p}} \mathcal{L}_{\alpha \beta}^{(p)} B^{(\beta) j_{1} \ldots j_{p}}\right] .
\end{aligned}
$$

This is invariant under the 'general coordinate transformation'

$$
\begin{aligned}
\delta A_{i_{1} \ldots i_{p}}^{(\alpha)}= & \xi^{j} \partial_{j} A_{i_{1} \ldots i_{p}}^{(\alpha)}+(-1)^{p-1}\left(\partial_{\left[i_{1}\right.} \xi^{j}\right) A_{\left.i_{2} \ldots i_{p}\right] j}^{(\alpha)} \\
& +\xi^{0}\left[(-1)^{p} \frac{g_{i_{1} j_{1} \ldots g} \ldots g_{i_{p} j_{p}}}{\sqrt{-g} g^{00}} \mathcal{L}_{\alpha \beta}^{(p)} B^{(\beta) j_{1} \ldots j_{p}}-\frac{g^{0 k}}{g^{00}} \partial_{[k} A_{\left.i_{1} \ldots i_{p}\right]}^{(\alpha)}\right]
\end{aligned}
$$

If we eliminate $A_{i_{1} \ldots i_{p}}^{(2)}$ from the action $(2.29)$ by its equation of motion, we get back the covariantized form of the action (2.27). Also, in this case the general coordinate transformation law of the field $A_{i_{1} \ldots i_{p}}^{(1)}$ reduces to the standard form in the $A_{0 i_{1} \ldots i_{p-1}}^{(1)}=0$ gauge. Finally, if we diagonalize the matrix $\mathcal{L}^{(p)}$, we get back the sum of the action of a self-dual $(p+1)$-form field strength and an anti-self-dual $(p+1)$-form field strength in curved space-time, as written down in ref.[20].

\section{4. $m$-FORM FiELdS IN $d$ Dimensions}

Let us consider next the free field theory of an $m$-form field $A_{\mu_{1} \ldots \mu_{m}}$ in $d$ dimensions. The corresponding field strength is

$$
F_{\mu_{1} \ldots \mu_{m+1}}=\partial_{\left[\mu_{1}\right.} A_{\left.\mu_{2} \ldots \mu_{m+1}\right]}, \quad 0 \leq \mu_{l} \leq d-1 .
$$


The equations of motion and the Bianchi identities are

$$
\eta^{\mu_{1} \rho_{1}} \partial_{\rho_{1}} F_{\mu_{1} \ldots \mu_{m+1}}=0, \quad \varepsilon^{\mu_{1} \ldots \mu_{m+2} \nu_{1} \ldots \nu_{d-m-2}} \partial_{\mu_{1}} F_{\mu_{2} \ldots \mu_{m+2}}=0 .
$$

We can dualize this theory by introducing a dual $(d-2-m)$-form potential, $B_{\nu_{1} \ldots \nu_{d-m-2}}$, and the corresponding field strength,

$$
G_{\nu_{1} \ldots \nu_{d-m-1}}=\partial_{\left[\nu_{1}\right.} B_{\left.\nu_{2} \ldots \nu_{d-m-1}\right]}
$$

such that

$$
\eta^{\mu_{1} \rho_{1}} \ldots \eta^{\mu_{m+1} \rho_{m+1}} F_{\rho_{1} \ldots \rho_{m+1}}=\frac{1}{(d-m-1) !} \varepsilon^{\mu_{1} \ldots \mu_{m+1} \nu_{1} \ldots \nu_{d-m-1}} G_{\nu_{1} \ldots \nu_{d-m-1}}
$$

It is easy to check that the equations of motion of $F$ correspond to Bianchi identities of $G$ and vice versa. Examples of such pairs of dual fields are a scalar and a two-form field in four dimensions, a two-form field and a six-form field in ten dimensions, etc.

Normally, the action of such a theory is written either in terms of the original field $A$ or the dual field $B$, but not both. We shall now write down a form of the action in which $A$ and $B$ appear on an equal footing. Consider the action

$$
\begin{aligned}
& S_{0}= \frac{1}{m !(d-m-1) !} \epsilon^{i_{1} \ldots i_{m} j_{1} \ldots j_{d-m-1}} F_{0 i_{1} \ldots i_{m}} G_{j_{1} \ldots j_{d-m-1}} \\
&+\frac{1}{2 \cdot(m+1) !} F_{i_{1} \ldots i_{m+1}} F_{i_{1} \ldots i_{m+1}} \\
&+\frac{1}{2 \cdot(d-m-1) !} G_{i_{1} \ldots i_{d-m-1}} G_{i_{1} \ldots i_{d-m-1}}, \\
& 1 \leq i_{l}, j_{l} \leq d .
\end{aligned}
$$

The action (2.35) is invariant under the following gauge transformations:

$$
\begin{aligned}
\delta A_{0 i_{1} \ldots i_{m-1}} & =\Psi_{i_{1} \ldots i_{m-1}}^{(1)}, \quad \delta B_{0 i_{1} \ldots i_{d-m-3}}=\Psi_{i_{1} \ldots i_{d-m-3}}^{(2)} \\
\delta A_{i_{1} \ldots i_{m}} & \left.=\partial_{\left[i_{1}\right.} \Lambda_{\left.i_{2} \ldots i_{m}\right]}^{(1)}, \quad \delta B_{i_{1} \ldots i_{d-m-2}}=\partial_{\left[i_{1}\right.} \Lambda_{i_{2} \ldots i_{d-m-2}}^{(2)}\right]
\end{aligned}
$$


Using the gauge transformation parameters $\Psi^{(1)}, \Psi^{(2)}$ we can set the gauge

$$
A_{0 i_{1} \ldots i_{m-1}}=0, \quad B_{0 i_{1} \ldots i_{d-m-3}}=0 .
$$

Finally, with the help of the gauge transformation parameters $\Lambda^{(1)}$ and $\Lambda^{(2)}$, the equations of motion derived from the action (2.35) can be shown to be precisely those given in eq.(2.34). Also, if we eliminate either the $A$ or the $B$ fields from the action (2.35) by their equations of motion, we get back the standard free action for the other field.

In many cases, the free equations (2.34) get modified by the addition of a Chern-Simons term to the field strength. The duality relations (2.34) then get modified to

$\eta^{\mu_{1} \rho_{1}} \ldots \eta^{\mu_{m+1} \rho_{m+1}}\left(F_{\rho_{1} \ldots \rho_{m+1}}+\Omega_{\rho_{1} \ldots \rho_{m+1}}\right)=\frac{1}{(d-m-1) !} \varepsilon^{\mu_{1} \ldots \mu_{m+1} \nu_{1} \ldots \nu_{d-m-1}} G_{\nu_{1} \ldots \nu_{d-m-1}}$,

where $\Omega$ is an $m+1$ form. We now ask the question: Is it possible to modify the action (2.35) in such a way that the corresponding equations of motion are the modified eqs.(2.38)? The answer to this question is yes. We simply need to add the term

$$
\begin{aligned}
S_{1}= & \frac{1}{(m+1) !} F_{i_{1} \ldots i_{m+1}} \Omega_{i_{1} \ldots i_{m+1}} \\
& +\frac{1}{m !} \frac{1}{(d-m-1) !} \epsilon^{i_{1} \ldots i_{m} j_{1} \ldots j_{d-m-1}} \Omega_{0 i_{1} \ldots i_{m}} G_{j_{1} \ldots j_{d-m-1}}
\end{aligned}
$$

to the action $S_{0}$ given in eq.(2.35).

\footnotetext{
$\star$ Note that although the addition of $\Omega$ to $F$ seems to destroy the symmetry between $F$ and $G$, we could have added the dual of $\Omega$ to $G$ with the same effect.
} 


\subsection{Supersymmetrization of the Duality-Invariant Maxwell AC-} TION

We shall now discuss how to supersymmetrize the duality-invariant Maxwell action while preserving manifest duality invariance. Since we are using a formalism that is not manifestly Lorentz invariant, we can use two-component spinors instead of four-component spinors for describing the fermionic fields in this theory. We know that the supersymmetry partner of a vector field in four dimensions should be a Majorana spinor. Such a field can be represented by a pair of complex twocomponent spinors $\psi^{(\alpha)}(1 \leq \alpha \leq 2)$ satisfying the condition ${ }^{\dagger}$

$$
\psi^{(\alpha) *}=\sigma_{2} \mathcal{L}_{\alpha \beta} \psi^{(\beta)} .
$$

Here $\sigma_{i}$ are the standard Pauli matrices. They act on the implicit spinor index of $\psi^{(\alpha)}$. The full action is now given by

$$
\begin{aligned}
S=\int d^{4} x[ & -\frac{1}{2}\left(B^{(\alpha) i} \mathcal{L}_{\alpha \beta} E_{i}^{(\beta)}+B^{(\alpha) i} B^{(\alpha) i}\right) \\
& \left.+i \psi^{(\alpha) \dagger} \partial_{0} \psi^{(\alpha)}-\psi^{(\alpha) \dagger} \mathcal{L}_{\alpha \beta} \sigma_{k} \partial_{k} \psi^{(\beta)}\right] .
\end{aligned}
$$

This action is invariant under the following supersymmetry transformations:

$$
\begin{aligned}
\delta \psi^{(\alpha)} & =\frac{1}{2}\left(\mathcal{L}_{\alpha \beta} \sigma_{k} B^{(\beta) k} \epsilon-\sigma_{k} B^{(\alpha) k} \sigma_{2} \epsilon^{*}\right) \\
\delta A_{i}^{(\alpha)} & =i \psi^{(\alpha) \dagger} \sigma_{i} \epsilon-i \psi^{(\beta) \dagger} \mathcal{L}_{\alpha \beta} \sigma_{i} \sigma_{2} \epsilon^{*},
\end{aligned}
$$

where $\epsilon$ is an arbitrary two-component complex spinor.

In order to see that the action (2.41) and the transformation laws (2.42) reduce to the standard action and supersymmetry transformation laws in four dimensions

\footnotetext{
$\dagger$ These are essentially the same thing as what is often described as two-component spinors with dotted and undotted indices. The notation used here is much more natural in the present context.
} 
when we eliminate the auxiliary fields $A_{i}^{(2)}$ by their equations of motion, we introduce four-component spinors

$$
\begin{gathered}
\psi=\left(\begin{array}{c}
\psi^{(1)} \\
\psi^{(2)}
\end{array}\right) \\
\eta=i\left(\begin{array}{c}
\sigma_{2} \epsilon^{*} \\
\epsilon
\end{array}\right)
\end{gathered}
$$

and the four-dimensional matrices $\gamma^{\mu}$ such that

$$
\gamma^{0} \gamma^{i}=\left(\begin{array}{cc}
0 & -i \sigma_{i} \\
i \sigma_{i} & 0
\end{array}\right)
$$

In terms of these quantities, the fermion bilinear term in eq.(2.41) may be written as

$$
-i \bar{\psi} \gamma \cdot \partial \psi
$$

Also, using eq.(2.8) the supersymmetry transformation laws given in eq.(2.42) may be rewritten as

$$
\delta A_{i}^{(1)}=i \bar{\psi} \gamma^{i} \eta, \quad \delta \psi=\frac{1}{4} \gamma_{\mu} \gamma_{\nu} F^{\mu \nu} \eta
$$

which are the standard supersymmetry transformation laws in four dimensions. Finally, from eqs.(2.40), (2.43) and (2.44) we see that $\psi$ and $\eta$ satisfy the Majorana condition

$$
\psi^{*}=i \gamma^{0} \gamma^{2} \psi, \quad \eta^{*}=i \gamma^{0} \gamma^{2} \eta
$$

Since the fermi terms in eq.(2.41) have been shown to agree with the standard formula for the kinetic term of a spinor, they can be coupled to gravity, thereby 
achieving general coordinate invariance and local Lorentz invariance, in the standard way, namely

$$
\int d^{4} x \sqrt{-g}\left[i e_{0}^{\mu} \psi^{(\alpha) \dagger} D_{\mu} \psi^{(\alpha)}-e_{k}^{\mu} \psi^{(\alpha) \dagger} \mathcal{L}_{\alpha \beta} \sigma_{k} D_{\mu} \psi^{(\beta)}\right]
$$

where $D_{\mu}$ denotes the covariant derivative involving the spin connection. The coupling to supergravity can then be worked out by standard methods.

\section{Low-Energy Effective Action in String Theory with Manifest SL $(2, R)$ Symmetry}

The low-energy effective action describing heterotic string theory compactified on a six-dimensional torus at a generic point in the moduli space is given by [9] [18]

$$
\begin{gathered}
\int d^{4} x \sqrt{-g}\left[R-\frac{1}{2\left(\lambda_{2}\right)^{2}} g^{\mu \nu} \partial_{\mu} \lambda \partial_{\nu} \bar{\lambda}-\frac{\lambda_{2}}{4} F_{\mu \nu}^{a}(L M L)_{a b} F^{b \mu \nu}\right. \\
\left.+\frac{\lambda_{1}}{4} F_{\mu \nu}^{a} L_{a b} \tilde{F}^{b \mu \nu}+\frac{1}{8} g^{\mu \nu} \operatorname{Tr}\left(\partial_{\mu} M L \partial_{\nu} M L\right)\right]
\end{gathered}
$$

where $A_{\mu}^{a}(1 \leq a \leq 28)$ are a set of 28 abelian gauge fields and

$$
\begin{gathered}
F_{\mu \nu}^{a}=\partial_{\mu} A_{\nu}^{a}-\partial_{\nu} A_{\mu}^{a}, \quad \tilde{F}^{a \mu \nu}=\frac{1}{2}(\sqrt{-g})^{-1} \epsilon^{\mu \nu \rho \sigma} F_{\rho \sigma}^{a} . \\
\lambda=\lambda_{1}+i \lambda_{2}
\end{gathered}
$$

is a complex scalar field,

$$
L=\left(\begin{array}{ccc}
0 & I_{6} & 0 \\
I_{6} & 0 & 0 \\
0 & 0 & -I_{16}
\end{array}\right)
$$

and $M$ is a $28 \times 28$ matrix-valued scalar field satisfying the constraints

$$
M^{T}=M, \quad M^{T} L M=L
$$


The action (3.1) is manifestly invariant under an $\mathrm{O}(6,22)$ transformation

$$
M \rightarrow \Omega^{T} M \Omega, \quad A_{\mu}^{a} \rightarrow \Omega_{a b}^{T} A_{\mu}^{b}
$$

where $\Omega$ is a $28 \times 28$ matrix satisfying

$$
\Omega^{T} L \Omega=L
$$

The equations of motion derived from the action (3.1) have a further SL(2,R) symmetry [8] [12] [13] [14], given by

$$
\lambda \rightarrow \frac{a \lambda+b}{c \lambda+d}, \quad F_{\mu \nu}^{a} \rightarrow c \lambda_{2}(M L)_{a b} \tilde{F}_{\mu \nu}^{b}+\left(c \lambda_{1}+d\right) F_{\mu \nu}^{a}, \quad a d-b c=1 .
$$

The action (3.1), however, is not invariant under this $\mathrm{SL}(2, \mathrm{R})$ transformation. More specifically, the terms involving the gauge fields are not invariant; the other terms are invariant.

In subsection (3.1) we shall show that, using the formalism of the previous section, we can write down a manifestly $\mathrm{SL}(2, \mathrm{R}) \times \mathrm{O}(6,22)$ invariant action, which is equivalent to the action (3.1). The price that we'll have to pay is again manifest general coordinate invariance of the action. We shall also see that SL(2,R) and $\mathrm{O}(6,22)$ transformations appear in a symmetric manner in the resulting action. The analysis of this subsection raises the question whether it is possible to write down a third form of the action in which $\mathrm{SL}(2, \mathrm{R})$ and general coordinate invariance of the action are manifest, but $\mathrm{O}(6,22)$ appears only as a symmetry of the equations of motion. In subsection (3.2) we show that this is possible for a restricted class of field configurations - the configurations for which all four-dimensional fields arising out of dimensional reduction of ten dimensional $\mathrm{U}(1)^{16}$ gauge fields are set to zero. 


\subsection{Manifestly $\operatorname{SL}(2, \mathrm{R}) \times \mathrm{O}(6,22)$ InVARIANT ACtion}

We shall carry out the construction of a manifestly $\mathrm{SL}(2, \mathrm{R}) \times \mathrm{O}(6,22)$ invariant action in three steps. In the first step we shall show how to generalize the action (2.1) to the case of multicomponent gauge fields. As we shall see, this will automatically introduce the matrix $M$ appearing in eq.(3.1) and satisfying (3.5) into the action. In the second step, we shall show how to couple the action (2.1) to the complex field $\lambda$ transforming as in eq.(3.8) in an $\mathrm{SL}(2, \mathrm{R})$ invariant manner. Finally, in the third step, we shall combine steps 1 and 2, as well as the result of the last section, to couple the gauge fields to the matrix-valued field $M$, the complex field $\lambda$, and the metric $g_{\mu \nu}$ in an SL(2,R) invariant fashion.

Step 1. We consider generalization of the action (2.1) to multicomponent gauge fields $A_{\mu}^{(a, \alpha)}$. The general form of the action consistent with the requirement of duality symmetry (2.10), gauge invariance (2.4), rotational invariance, and invariance under the parity transformation $A_{i}^{(a, \alpha)}\left(x^{0}, \vec{x}\right) \rightarrow(-1)^{\alpha} A_{i}^{(a, \alpha)}\left(x^{0},-\vec{x}\right)$, is given by ${ }^{\star}$

$$
S_{P, Q}=-\frac{1}{2} \int d^{4} x\left[B^{(a, \alpha) i} \mathcal{L}_{\alpha \beta} Q_{a b} E_{i}^{(b, \beta)}+B^{(a, \alpha) i} P_{a b} B^{(b, \alpha) i}\right],
$$

where $Q$ is a space-time independent matrix, $P$ is a space-time dependent matrix (in general), and

$$
B^{(a, \alpha) i}=\epsilon^{i j k} \partial_{j} A_{k}^{(a, \alpha)}, \quad E_{i}^{(a, \alpha)}=\partial_{0} A_{i}^{(a, \alpha)}-\partial_{i} A_{0}^{(a, \alpha)}
$$

Since only the symmetric parts of $Q$ and $P$ contribute to the action, we can choose these matrices to be symmetric without any loss of generality. Also, using the freedom of a linear redefinition of the gauge fields, $A_{i}^{(a, \alpha)} \rightarrow S_{a b} A_{i}^{(b, \alpha)}$, where $S$ is a space-time independent matrix, we can ensure that the matrix $Q$ has eigenvalues

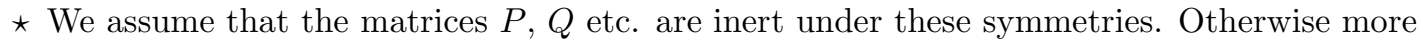
general possibilities may arise.
} 
\pm 1 , so that $Q^{2}=I$. If we now eliminate the fields $A_{i}^{(a, 2)}$ from the action (3.9) using their equations of motion, we get the action

$$
-\frac{1}{2} \int d^{4} x\left[B^{(a, 1) i} P_{a b} B^{(b, 1) i}-E_{i}^{(a, 1)}\left(Q P^{-1} Q\right)_{a b} E_{i}^{(b, 1)}\right]
$$

This action is manifestly Lorentz invariant provided

$$
Q P^{-1} Q=P
$$

Comparing eqs.(3.11), (3.12), with (3.1), (3.5) in the background $g_{\mu \nu}=\eta_{\mu \nu}, \lambda=i$, we see that we need the identification

$$
Q=L, \quad P=L M L
$$

The action (3.9) is not manifestly Lorentz invariant. But it is invariant under hidden Lorentz transformations, which are direct generalizations of the Lorentz transformation laws (2.12). Since these transformation laws can always be derived from the general coordinate transformation laws of the final action that we shall write down, we shall not write down the Lorentz transformation laws of the fields $A_{i}^{(a, \alpha)}$ explicitly here.

Step 2. We now go back to the action (2.1) and try to couple the complex field $\lambda$ to this action in an $\mathrm{SL}(2, \mathrm{R})$ invariant fashion. In order to do this, we first introduce a matrix

$$
\mathcal{M}(\lambda)=\frac{1}{\lambda_{2}}\left(\begin{array}{cc}
1 & \lambda_{1} \\
\lambda_{1} & |\lambda|^{2}
\end{array}\right)
$$

satisfying,

$$
\mathcal{M}^{T}=\mathcal{M}, \quad \mathcal{M L} \mathcal{M}^{T}=\mathcal{L}
$$

Under the $\mathrm{SL}(2, \mathrm{R})$ transformation (3.8) of the field $\lambda$, the matrix $\mathcal{M}$ transforms 
in a simple manner,

$$
\mathcal{M} \rightarrow \omega^{T} \mathcal{M} \omega
$$

where

$$
\omega=\left(\begin{array}{ll}
d & b \\
c & a
\end{array}\right) .
$$

An $\mathrm{SL}(2, \mathrm{R})$ invariant coupling of the action (2.1) to the field $\lambda$ may now be written down as follows:

$$
S_{\lambda}=-\frac{1}{2} \int d^{4} x\left[B^{(\alpha) i} \mathcal{L}_{\alpha \beta} E_{i}^{(\beta)}+B^{(\alpha) i}\left(\mathcal{L}^{T} \mathcal{M} \mathcal{L}\right)_{\alpha \beta} B^{(\beta) i}\right]
$$

Using the relation

$$
\omega \mathcal{L} \omega^{T}=\mathcal{L}
$$

one can easily see that the action (3.18) is invariant under the transformation (3.16) on $\mathcal{M}$, together with the transformation

$$
A_{i}^{(\alpha)} \rightarrow\left(\omega^{T}\right)_{\alpha \beta} A_{i}^{(\beta)}
$$

After eliminating the fields $A_{i}^{(2)}$ using their equations of motion, we get the action

$$
-\frac{1}{4} \int d^{4} x\left(\lambda_{2} F_{\mu \nu}^{(1)} F_{\rho \sigma}^{(1)}-\lambda_{1} F_{\mu \nu}^{(1)} \tilde{F}_{\rho \sigma}^{(1)}\right) \eta^{\mu \rho} \eta^{\nu \sigma}
$$

The gauge field dependent part of the action (3.1) in flat background, and for $M=I, L=I$, is precisely 22 copies of this action. Also, the duality transformation (3.20) takes precisely the form of eq.(3.8) with $M=L=I$ after we eliminate $A_{i}^{(2)}$ from these transformation laws using their equations of motion. The gauge fields $A_{\mu}^{(2)}$ may be identified with the dual vector potentials introduced by Kallosh and Ortin [23].

Again, the action (3.18) has a hidden Lorentz invariance. But we shall not write down the Lorentz transformation laws of the gauge fields explicitly here. 
Step 3. We shall now combine eqs.(2.16), (3.18) and (3.9) together, for the identification given in eq.(3.13), to obtain the manifestly $\mathrm{SL}(2, \mathrm{R})$ invariant coupling of the gauge fields $A_{\mu}^{(a, \alpha)}$ to the fields $M, \lambda$ and $g_{\mu \nu}$. The resulting action is

$$
\begin{gathered}
S_{\lambda, M, g}=-\frac{1}{2} \int d^{4} x\left[B^{(a, \alpha) i} \mathcal{L}_{\alpha \beta} L_{a b} E_{i}^{(b, \beta)}+\epsilon^{i j k} \frac{g^{0 k}}{g^{00}} B^{(a, \alpha) i} \mathcal{L}_{\alpha \beta} L_{a b} B^{(b, \beta) j}\right. \\
\left.-\frac{g_{i j}}{\sqrt{-g} g^{00}} B^{(a, \alpha) i}\left(\mathcal{L}^{T} \mathcal{M L}\right)_{\alpha \beta}(L M L)_{a b} B^{(b, \beta) j}\right]
\end{gathered}
$$

If we eliminate the fields $A_{i}^{(a, 2)}$ from this action using their equations of motion, we get back the gauge field dependent part of the action (3.1)

$$
-\frac{1}{4} \int d^{4} x \sqrt{-g}\left[\lambda_{2} F_{\mu \nu}^{(a, 1)}(L M L)_{a b} F^{(b, 1) \mu \nu}-\lambda_{1} F_{\mu \nu}^{(a, 1)} L_{a b} \tilde{F}^{(b, 1) \mu \nu}\right] .
$$

The action (3.22) is manifestly invariant under the $\mathrm{O}(6,22)$ transformation given in eqs.(3.7) and the $\mathrm{SL}(2, \mathrm{R})$ transformation given in eqs.(3.16), (3.20). It is not manifestly invariant under general coordinate transformations. However, it can be checked that it is invariant under the transformation

$$
\begin{aligned}
\delta A_{i}^{(a, \alpha)}= & \xi^{j} \partial_{j} A_{i}^{(a, \alpha)}+\left(\partial_{i} \xi^{j}\right) A_{j}^{(a, \alpha)} \\
& -\xi^{0}\left\{\frac{g_{i j}}{\sqrt{-g} g^{00}}(\mathcal{M L})_{\alpha \beta}(M L)_{a b} B^{(b, \beta) j}+\frac{g^{0 k}}{g^{00}} \epsilon^{i j k} B^{(a, \alpha) j}\right\},
\end{aligned}
$$

which generalizes (2.17) and reduces to the usual general coordinate transformation law of the field $A_{i}^{(a, 1)}$ in the $A_{0}^{(a, 1)}=0$ gauge when the fields $A_{i}^{(a, 2)}$ are eliminated by their equations of motion.

In terms of the matrix $\mathcal{M}$, the $\lambda$ field kinetic term appearing in eq.(3.1) can also be written in a manifestly $\mathrm{SL}(2, \mathrm{R})$ invariant form:

$$
\frac{1}{2\left(\lambda_{2}\right)^{2}} g^{\mu \nu} \partial_{\mu} \lambda \partial_{\nu} \bar{\lambda}=\frac{1}{4} g^{\mu \nu} \operatorname{tr}\left(\partial_{\mu} \mathcal{M} \mathcal{L} \partial_{\nu} \mathcal{M} \mathcal{L}\right)
$$


Thus the full action (3.1) may be replaced by

$$
\begin{aligned}
S=\int d^{4} x[ & \sqrt{-g}\left\{R-\frac{1}{4} g^{\mu \nu} \operatorname{tr}\left(\partial_{\mu} \mathcal{M L} \partial_{\nu} \mathcal{M L}\right)+\frac{1}{8} g^{\mu \nu} \operatorname{Tr}\left(\partial_{\mu} M L \partial_{\nu} M L\right)\right\} \\
& -\frac{1}{2}\left\{B^{(a, \alpha) i} \mathcal{L}_{\alpha \beta} L_{a b} E_{i}^{(b, \beta)}+\epsilon^{i j k} \frac{g^{0 k}}{g^{00}} B^{(a, \alpha) i} \mathcal{L}_{\alpha \beta} L_{a b} B^{(b, \beta) j}\right. \\
& \left.\left.-\frac{g_{i j}}{\sqrt{-g} g^{00}} B^{(a, \alpha) i}\left(\mathcal{L}^{T} \mathcal{M L}\right)_{\alpha \beta}(L M L)_{a b} B^{(b, \beta) j}\right\}\right]
\end{aligned}
$$

In the above equation $\operatorname{Tr}$ denotes trace over the indices $a, b$ and $\operatorname{tr}$ denotes trace over the indices $\alpha, \beta$. Note that the matrices $\mathcal{M}, \mathcal{L}$ and $M, L$ appear quite symmetrically in the expression for $S$.

In three dimensions both $\mathrm{SL}(2, \mathrm{R})$ and $\mathrm{O}(6,22)$ become part of a larger symmetry group $\mathrm{O}(8,24)[24]$. This provides further evidence that $\mathrm{SL}(2, \mathrm{R})$ and $\mathrm{O}(6,22)$ should play identical roles in the full string theory.

\subsection{Action With Manifest SL(2,R) and General Coordinate In-} VARIANCE

We have seen that starting with the action (3.26) and eliminating the auxiliary fields $A_{i}^{(a, 2)}$ by their equations of motion gave the manifestly $O(6,22)$ and general coordinate invariant action (3.1). Note, however, that in the action (3.26) the various fields $A_{\mu}^{(a, \alpha)}$ appear symmetrically, and hence it is a matter of choice which subset of these fields we treat as auxiliary fields. If we choose the subset of auxiliary fields to be invariant under $\mathrm{O}(6,22)$ transformations, then we would expect the final action to be manifestly invariant under $\mathrm{O}(6,22)$ transformations, as was the case in going from the action (3.26) to (3.1). But the same argument shows that if we choose the set of auxiliary fields in such a way that the set is invariant under $\mathrm{SL}(2, \mathrm{R})$ transformations, then the resulting action should be manifestly $\mathrm{SL}(2, \mathrm{R})$ invariant, but not manifestly $\mathrm{O}(6,22)$ invariant. This naturally gives rise to the question as to whether it is possible to get a manifestly $\mathrm{SL}(2, \mathrm{R})$ and general coordinate invariant action following this procedure. 
We shall now show that it is possible to obtain such an action provided we set all the fields arising from the dimensional reduction of ten-dimensional gauge fields to zero. In terms of the fields appearing in eq.(3.1) this means that we now take the gauge fields to have 12 components instead of 28 components, $L$ to be the $12 \times 12$ matrix

$$
L=\left(\begin{array}{cc}
0 & I_{6} \\
I_{6} & 0
\end{array}\right)
$$

and $M$ to be a $12 \times 12$ matrix-valued field satisfying the same constraints (3.5) with respect to the new $L$. Such a matrix $M$ can be parametrized as

$$
M=\left(\begin{array}{cc}
\hat{G}^{-1} & \hat{G}^{-1} \hat{B} \\
-\hat{B} \hat{G}^{-1} & \hat{G}-\hat{B} \hat{G}^{-1} \hat{B}
\end{array}\right),
$$

where $\hat{G}$ and $\hat{B}$ are $6 \times 6$ symmetric and antisymmetric matrices, respectively, which can be identified with the internal components of the ten-dimensional metric and antisymmetric tensor fields, respectively. The $\mathrm{O}(6,6) \times \mathrm{SL}(2, \mathrm{R})$ invariant form of the action is given by eq.(3.26) with the indices $a, b$ running from 1 to 12 .

We now start from eq.(3.26) and eliminate the fields $A_{i}^{(m+6, \alpha)}(1 \leq m \leq 6$, $1 \leq \alpha \leq 2)$ by their equations of motion. With appropriate choice of gauge, these equations can be brought to the form:

$$
\begin{aligned}
g_{i j} B^{(m+6, \alpha) j}= & -\sqrt{-g} g^{00} \hat{G}_{m n}\left(\mathcal{M} \mathcal{L}^{T}\right)_{\alpha \beta}\left\{E_{i}^{(n, \beta)}+\epsilon^{i j k} \frac{g^{0 k}}{g^{00}} B^{(n, \beta) j}\right\} \\
& -g_{i j} \hat{B}_{m n} B^{(n, \alpha) j} .
\end{aligned}
$$

Here $i, j, k$ are spatial indices, and $m, n$ are indices denoting the six internal directions. If we now substitute this back into the action (3.26), we get an action of the

\footnotetext{
* Note that this is an $\mathrm{SL}(2, \mathrm{R})$ invariant set.
} 
form:

$$
\begin{aligned}
& \int d^{4} x \sqrt{-g}\left[R-\frac{1}{4} g^{\mu \nu} \operatorname{tr}\left(\partial_{\mu} \mathcal{M} \mathcal{L} \partial_{\nu} \mathcal{M L}\right)+\frac{1}{8} g^{\mu \nu} \operatorname{Tr}\left(\partial_{\mu} M L \partial_{\nu} M L\right)\right. \\
& \left.-\frac{1}{4} F_{\mu \nu}^{(m, \alpha)} \hat{G}_{m n}\left(\mathcal{L}^{T} \mathcal{M L}\right)_{\alpha \beta} F_{\rho \sigma}^{(n, \beta)} g^{\mu \rho} g^{\nu \sigma}-\frac{1}{4} F_{\mu \nu}^{(m, \alpha)} \hat{B}_{m n} \mathcal{L}_{\alpha \beta} \tilde{F}_{\rho \sigma}^{(n, \beta)} g^{\mu \rho} g^{\nu \sigma}\right] .
\end{aligned}
$$

This action is manifestly $\mathrm{SL}(2, \mathrm{R})$ and Lorentz invariant, but not manifestly $\mathrm{O}(6,6)$ invariant. However, since the equations of motion derived from this action are identical to those derived from the action (3.26), we can conclude that these equations of motion are also $\mathrm{O}(6,6)$ invariant.

\section{Manifestly $\mathrm{SL}(2, \mathrm{R})$ Invariant Effective Action from Dimensional Reduction of $N=1 D=10$ Supergravity Theory}

In the previous section we have given a formulation of the low-energy effective action in heterotic string theory that is manifestly $\mathrm{SL}(2, \mathrm{R})$ and $\mathrm{O}(6,22)$ invariant, but not manifestly general coordinate invariant. We have also shown that in the special case where all the components of the ten-dimensional gauge fields are set to zero, we can get a manifestly $\mathrm{SL}(2, \mathrm{R})$ and general coordinate invariant action by sacrificing $\mathrm{O}(6,22)$ invariance. This analysis puts the $\mathrm{O}(6,22)$ and $\mathrm{SL}(2, \mathrm{R})$ symmetry on an equal footing from the point of view of the four-dimensional effective field theory. However, it is the manifestly $\mathrm{O}(6,22)$ and general coordinate invariant form of the action that arises naturally in the dimensional reduction of the $N=1$ supergravity theory in ten dimensions. From this point of view, the $\mathrm{O}(6,22)$ symmetry of the action might appear to be more fundamental than the $\operatorname{SL}(2, \mathrm{R})$ symmetry. In this section we shall get rid of this asymmetry by showing that it is the $\mathrm{SL}(2, \mathrm{R})$ invariant action (3.30) that arises naturally in the dimensional reduction of another ten-dimensional theory - the dual formulation of the $N=1$ $D=10$ supergravity theory [25].

Before we can write down the field content and action of this ten-dimensional theory, we must describe our notation. We shall denote ten-dimensional coordinates by $z^{M}(0 \leq M \leq 9)$, whereas $y^{m}(4 \leq m \leq 9)$ and $x^{\mu}(0 \leq \mu \leq 3)$ will 
denote the internal and space-time coordinates, respectively. The superscript (10) will denote fields that appear naturally in the ten-dimensional theory; the fields which are more natural from the point of view of four-dimensional theory will not carry this superscript. The subscript $S$ will denote the metric which couples naturally to the string (the one that appears in the world-sheet action). Finally, since the fields appearing in the dual formulation of the $N=1 D=10$ supergravity theory couple naturally to the five-brane [3] [4], it is also convenient to introduce a new metric that couples naturally to the five-brane; we shall denote this one by the subscript $F$.

In the absence of ten-dimensional gauge fields, the only bosonic fields in the dual formulation of the $N=1$ supergravity theory in 10 dimensions are the metric $G_{F M N}^{(10)}$, the dilaton $\Phi^{(10)}$ and the 6 -form field $\mathcal{A}_{M_{1} \ldots M_{6}}^{(10)}$. The bosonic part of the action may be written as [5]

$$
\begin{aligned}
S=\int d^{10} z & \sqrt{-\operatorname{det} G_{F}^{(10)}} e^{\Phi^{(10)} / 3}\left(R_{F}^{(10)}\right. \\
& \left.-\frac{1}{2.7 !} G_{F}^{(10) M_{1} N_{1}} \ldots G_{F}^{(10) M_{7} N_{7}} K_{M_{1} \ldots M_{7}}^{(10)} K_{N_{1} \ldots N_{7}}^{(10)}\right)
\end{aligned}
$$

where

$$
K_{M_{1} \ldots M_{7}}^{(10)}=\partial_{\left[M_{1}\right.} \mathcal{A}_{\left.M_{2} \ldots M_{7}\right]}^{(10)}
$$

The string metric $G_{S M N}^{(10)}$ is related to the five-brane metric metric $G_{F M N}^{(10)}$ through the relation [5]

$$
G_{F M N}^{(10)}=e^{-\Phi^{(10)} / 3} G_{S M N}^{(10)}
$$

In terms of the metric $G_{S M N}^{(10)}$, the first term in the action (4.1) may be written as $[5]$

$$
\begin{aligned}
S_{1} & \equiv \int d^{10} z \sqrt{-\operatorname{det} G_{F}^{(10)}} e^{\Phi^{(10)} / 3} R_{F}^{(10)} \\
& =\int d^{10} z \sqrt{-\operatorname{det} G_{S}^{(10)}} e^{-\Phi^{(10)}}\left(R_{S}^{(10)}+G_{S}^{(10) M N} \partial_{M} \Phi^{(10)} \partial_{N} \Phi^{(10)}\right)
\end{aligned}
$$

Dimensional reduction of this term to four dimensions was already carried out in 
ref.[9], so we just state the results here. We define [9] [13]

$$
\begin{aligned}
\hat{G}_{m n} & =G_{S m n}^{(10)}, \quad C_{\mu}^{m}=\hat{G}^{m n} G_{S n \mu}^{(10)}, \quad G_{S \mu \nu}=G_{S \mu \nu}^{(10)}-G_{S m \mu}^{(10)} G_{S n \nu}^{(10)} \hat{G}^{m n} \\
\Phi & =\Phi^{(10)}-\frac{1}{2} \ln \operatorname{det} \hat{G}, \quad \lambda_{2}=e^{-\Phi}, \quad g_{\mu \nu}=e^{-\Phi} G_{S \mu \nu} .
\end{aligned}
$$

In the above equations $\hat{G}^{m n}$ denotes the matrix inverse of $\hat{G}_{m n}$. If we take the various fields to be independent of the internal coordinates, and normalize $\int d^{6} y$ to 1 , we get the following form of the dimensionally reduced action:

$$
\begin{aligned}
S_{1}= & \int d^{4} x \sqrt{-g}\left[R-\frac{1}{2\left(\lambda_{2}\right)^{2}} g^{\mu \nu} \partial_{\mu} \lambda_{2} \partial_{\nu} \lambda_{2}+\frac{1}{4} g^{\mu \nu} \operatorname{Tr}\left(\partial_{\mu} \hat{G} \partial_{\nu} \hat{G}^{-1}\right)\right. \\
& \left.-\frac{1}{4} \lambda_{2} \hat{G}_{m n} g^{\mu \rho} g^{\nu \sigma} F_{\mu \nu}^{(C) m} F_{\rho \sigma}^{(C) n}\right],
\end{aligned}
$$

where

$$
F_{\mu \nu}^{(C) m}=\partial_{\mu} C_{\nu}^{m}-\partial_{\nu} C_{\mu}^{m}
$$

We now need to carry out the dimensional reduction of the second term in the action (4.1). This has been carried out in detail in the appendix; here we only quote the result. The final result agrees with the action (3.30), provided we make the identifications

$$
\begin{aligned}
\lambda_{1} & =\frac{1}{6 !} \epsilon^{m_{1} \ldots m_{6}} \mathcal{A}_{m_{1} \ldots m_{6}}^{(10)} \\
A_{\mu}^{(m, 1)} & =C_{\mu}^{m} \\
A_{\mu}^{(m, 2)} & =\frac{1}{5 !} \epsilon^{m m_{2} \ldots m_{6}}\left(\mathcal{A}_{\mu m_{2} \ldots m_{6}}^{(10)}-C_{\mu}^{n} \mathcal{A}_{n m_{2} \ldots m_{6}}^{(10)}\right)
\end{aligned}
$$

and $\hat{B}_{m_{1} m_{2}}$ to the duals of the antisymmetric tensor fields

$$
\begin{aligned}
\mathcal{B}_{\nu \rho}^{\left(m_{1} m_{2}\right)}= & \frac{1}{4 !} \epsilon^{m_{1} \ldots m_{6}} \mathcal{A}_{\nu \rho m_{3} \ldots m_{6}}^{(10)} \\
& -\left[\left(\lambda_{1} C_{\nu}^{m_{1}} C_{\rho}^{m_{2}}+\frac{1}{2} \mathcal{D}_{\nu}^{m_{1}} C_{\rho}^{m_{2}}-\frac{1}{2} \mathcal{D}_{\rho}^{m_{1}} C_{\nu}^{m_{2}}\right)-\left(m_{1} \leftrightarrow m_{2}\right)\right]
\end{aligned}
$$

This analysis shows that the $\mathrm{SL}(2, \mathrm{R})$ invariance appears naturally in the dimensional reduction of the dual form of the $N=1$ supergravity theory in ten dimensions. Since the fields appearing in this form of the supergravity theory couple 
naturally to the five-brane, this prompts us to speculate that the $\mathrm{SL}(2, \mathrm{R})$ transformation plays the same role in the theory of five-branes as the $\mathrm{O}(6,22)$ transformation in the theory of strings. The conjecture that the discrete $\mathrm{SL}(2, \mathrm{Z})$ subgroup of $\mathrm{SL}(2, \mathrm{R})$ is an exact symmetry of string theory [15] [13] [18] [14] [19] suggests that it is an exact symmetry of the five-brane spectrum and interactions, with the Kaluza-Klein modes and the five-brane winding modes getting interchanged under the duality transformation. In order to test this conjecture, however, it would be helpful to know the full spectrum of the five-brane theory.

The similarity between the usual $R \rightarrow 1 / R$ duality transformation and the coupling constant duality transformation may be made more explicit by expressing the complex field $\lambda$ in terms of the variables of the dual theory. If we define

$$
\hat{G}_{F m n}=G_{F m n}^{(10)}
$$

then from eqs.(4.3), (4.5) we get

$$
\operatorname{det} \hat{G}_{F}=e^{-2 \Phi^{(10)}} \operatorname{det} \hat{G}=e^{-2 \Phi} .
$$

This gives

$$
\lambda_{2}=\sqrt{\operatorname{det} \hat{G}_{F}} .
$$

Combining with the first of eqs.(4.8) this gives

$$
\lambda \equiv \lambda_{1}+i \lambda_{2}=\mathcal{A}_{1 \ldots 6}^{(10)}+i \sqrt{\operatorname{det} \hat{G}_{F}} .
$$

This expression is remarkably similar to the expression for the complex field that transforms in a similar fashion under the usual target space $\mathrm{SL}(2, \mathrm{Z})$ duality for heterotic string compactified on a two torus:

$$
T=B_{12}^{(10)}+i \sqrt{\operatorname{det} \hat{G}}
$$

Our proposal fits in naturally with the observation [6] that the roles of the $\sigma$-model loop expansion parameter and the string loop expansion parameter get 
interchanged in going from the string description of the theory to the five-brane description. Another related observation was made in ref.[26], where it was found that the magnetic monopole solutions in four-dimensional heterotic string theory, which are crucial for the $\mathrm{SL}(2, \mathrm{Z})$ invariance of the spectrum, may be constructed by wrapping the five-brane soliton solutions in this theory around the six-dimensional torus.

\section{Summary and Discussion}

In this paper we have shown that the low-energy effective action of toroidally compactified heterotic string theory can be written in a form that exhibits manifest $\mathrm{O}(6,22) \times \mathrm{SL}(2, \mathrm{R})$ symmetry. The resulting action is not manifestly general coordinate invariant, but does possess a symmetry that reduces to the standard general coordinate transformation laws when the auxiliary fields are eliminated by their equations of motion. We have also been able to get a manifestly $\operatorname{SL}(2, \mathrm{R})$ and general coordinate invariant effective action for a restricted class of field configurations in which all four-dimensional fields arising from the dimensional reduction of the $\mathrm{U}(1)^{16}$ gauge fields in ten dimensions are set to zero. This $\mathrm{SL}(2, \mathrm{R})$ and general coordinate invariant form of the action was shown to originate from the dimensional reduction of the dual formulation of the $N=1$ supergravity theory in ten dimensions without the gauge fields.

The analysis of this paper shows that the $\mathrm{O}(6,22)$ and $\mathrm{SL}(2, \mathrm{R})$ symmetries appear on an equal footing from the point of view of four-dimensional effective field theory. Since the discrete subgroup $\mathrm{O}(6,22 ; \mathrm{Z})$ is known to be an exact symmetry of (perturbative) string theory, this increases our confidence in the conjecture that the discrete $\mathrm{SL}(2, \mathrm{Z})$ subgroup of $\mathrm{SL}(2, \mathrm{R})$ might also be an exact symmetry of the theory. However, since SL $(2, R)$ arises naturally in the dual formulation of the ten-

dimensional supergravity theory, to have the $\mathrm{SL}(2, \mathrm{Z})$ symmetry manifest, we may need to go to a dual formulation of the theory - perhaps the theory of five-branes. 
One of the unsatisfactory features of our analysis has been that we had to ignore the $\mathrm{U}(1)^{16}$ gauge fields in ten dimensions to see an $\mathrm{SL}(2, \mathrm{R})$ invariant action come out of dimensional reduction of a ten-dimensional theory. However, since from the four-dimensional point of view we know that a manifestly $\mathrm{SL}(2, \mathrm{R})$ invariant action of the theory exists, one would suspect that there should be some formulation of the $N=1$ supergravity theory in ten dimensions coupled to abelian gauge fields, which, upon dimensional reduction, gives rise to a manifestly $\mathrm{SL}(2, \mathrm{R})$ invariant action. Such an action would probably provide a good starting point for the search for an alternative formulation of the theory in which the $\mathrm{SL}(2, \mathrm{Z})$ symmetry of the spectrum is manifest.

The analysis of sect.(2.4) already provides a clue as to what this new formulation of the ten-dimensional supergravity theory might be. From the analysis of sect.3, we have seen that the action with manifest $\mathrm{SL}(2, \mathrm{R})$ symmetry requires doubling of at least those gauge field components that arise from the $\mathrm{U}(1)^{16}$ gauge fields in ten dimensions. (For the gauge fields that arise from the ten-dimensional metric and the antisymmetric tensor fields, we can avoid the doubling, and at the same time, maintain manifest $\mathrm{SL}(2, \mathrm{R})$ invariance, by following the same procedure that took us from eq.(3.26) to eq.(3.30).) This would mean that we must have doubling of gauge fields in ten dimensions also. This, in turn, can be implemented by following the procedure given in sect.(2.4). Besides the $16 U(1)$ gauge fields $A_{M}^{I}$, we shall now also have 167 -form fields $\tilde{A}_{M_{1} \ldots M_{7}}^{I}$. Upon dimensional reduction, the fields $A_{M}^{I}$ gives rise to scalars $A_{m}^{I}$ and vectors $A_{\mu}^{I}$. The fields $\tilde{A}$ give rise to vectors $\tilde{A}_{1 \ldots 6 \mu}^{I}$, which provides the necessary doubling of the $16 \mathrm{U}(1)$ gauge fields in four dimensions. It also gives rise to antisymmetric tensor fields $\tilde{A}_{m_{1} \ldots m_{5} \mu \nu}$, which are dual to the scalar fields $A_{m}^{I}$ in the sense of sect.2.4. Thus the scalar fields $A_{m}^{I}$ (which form part of the matrix $M$ ) now appear in the formalism of sect.2.4. Although this destroys manifest $\mathrm{O}(6,22)$ symmetry, it does not destroy manifest $\mathrm{SL}(2, \mathrm{R})$ symmetry, since the fields $A_{m}^{I}$ (and hence also their duals) are $\mathrm{SL}(2, \mathrm{R})$ neutral. Finally, there are also $p$-form fields with $p \geq 3$ which appear from the dimensional reduction of the fields $\tilde{A}$, but in four dimensions these fields have no 
dynamics.

The question that one needs to address is whether there is a formulation of $N=1$ supergravity theory coupled to abelian gauge fields that naturally incorporates the action (2.35) for gauge fields in ten dimensions. The analysis of sect.2.5 provides a first step towards this formulation. If there is such a formalism, then the next question to ask would be if this formulation of the supergravity theory arises naturally from some other fundamental theory, possibly the five-brane, or some generalization.

Our discussion has focussed on generic points in moduli space, where all the gauge symmetries of the low-energy effective action are abelian. It would be nice to extend our analysis to deal with non-abelian gauge groups. This is a challenging problem, whose solution should be very enlightening. Finally, let us remark that the tools that we have introduced can be used to construct several theories that have been sought unsuccessfully in the past. One example is a reformulation of $\mathrm{N}=8 \mathrm{D}=4$ supergravity with the noncompact $E_{7,7}$ global symmetry realized as a symmetry of the action. In the usual formulation this symmetry rotates abelian gauge field strengths into their duals, just as in the case of $\operatorname{SL}(2, \mathrm{R})$ symmetry that we have presented. Another example is the construction of an action for type IIB supergravity in ten dimensions. Here the problem is the presence of a four-form potential with a self-dual five-form field strength. As we have seen, this can also be described by an action that sacrifices manifest covariance.

Acknowledgements: We would like to thank M. Duff, J. Harvey, C. Hull, A. Strominger and P. Townsend for useful discussions. 


\section{APPENDIX}

In this appendix we shall discuss the dimensional reduction of the second term in the action (4.1). We define the following four-dimensional fields in terms of the components of the six-form potential $\mathcal{A}^{(10)}$ in ten dimensions:

$$
\begin{aligned}
\lambda_{1}= & \frac{1}{6 !} \epsilon^{m_{1} \ldots m_{6}} \mathcal{A}_{m_{1} \ldots m_{6}}^{(10)} \\
\mathcal{D}_{\mu}^{m_{1}}= & \frac{1}{5 !} \epsilon^{m_{1} \ldots m_{6}}\left(\mathcal{A}_{\mu m_{2} \ldots m_{6}}^{(10)}-C_{\mu}^{m} \mathcal{A}_{m m_{2} \ldots m_{6}}^{(10)}\right) \\
\mathcal{B}_{\nu \rho}^{\left(m_{1} m_{2}\right)}= & \frac{1}{4 !} \epsilon^{m_{1} \ldots m_{6}} \mathcal{A}_{\nu \rho m_{3} \ldots m_{6}}^{(10)} \\
& -\left[\left(\lambda_{1} C_{\nu}^{m_{1}} C_{\rho}^{m_{2}}+\frac{1}{2} \mathcal{D}_{\nu}^{m_{1}} C_{\rho}^{m_{2}}-\frac{1}{2} \mathcal{D}_{\rho}^{m_{1}} C_{\nu}^{m_{2}}\right)-\left(m_{1} \leftrightarrow m_{2}\right)\right] \\
\mathcal{C}_{\nu \rho \sigma}^{m_{1} m_{2} m_{3}}= & \frac{1}{3 !} \epsilon^{m_{1} \ldots m_{6}} \mathcal{A}_{\nu \rho \sigma m_{4} \ldots m_{6}}^{(10)} .
\end{aligned}
$$

In terms of these fields, we define four-dimensional field strengths as follows:

$$
\begin{gathered}
F_{\mu \nu}^{(\mathcal{D}) m}=\partial_{\mu} \mathcal{D}_{\nu}^{m}-\partial_{\nu} \mathcal{D}_{\mu}^{m} \\
\hat{K}_{\mu \nu \rho}^{m_{1} m_{2}}=\left[\partial_{\mu} \mathcal{B}_{\nu \rho}^{m_{1} m_{2}}-\frac{1}{2}\left\{\left(C_{\rho}^{m_{2}} F_{\mu \nu}^{(\mathcal{D}) m_{1}}+\mathcal{D}_{\rho}^{m_{2}} F_{\mu \nu}^{(C) m_{1}}\right)-\left(m_{1} \leftrightarrow m_{2}\right)\right\}\right] \\
+ \text { cyclic permutations of } \mu, \nu, \rho
\end{gathered}
$$

and

$$
\begin{aligned}
\mathcal{K}_{\mu \nu \rho \sigma}^{m_{1} m_{2} m_{3}}= & {\left[\partial_{\mu} \mathcal{C}_{\nu \rho \sigma}^{m_{1} m_{2} m_{3}}+(-1)^{P} \cdot \text { cyclic permutations of } \mu, \nu, \rho, \sigma\right] } \\
& -\left[\left(C_{\sigma}^{m_{3}} \hat{K}_{\mu \nu \rho}^{m_{1} m_{2}}+\text { cyclic permutations of } m_{1}, m_{2}, m_{3}\right)\right. \\
& \left.+(-1)^{P} \cdot \text { cyclic permutations of } \mu, \nu, \rho, \sigma\right] \\
& -\left[\left\{C_{\sigma}^{m_{3}} C_{\rho}^{m_{2}}\left(F_{\mu \nu}^{(\mathcal{D}) m_{1}}+\lambda_{1} F_{\mu \nu}^{(C) m_{1}}\right)\right.\right. \\
& \left.+(-1)^{P} \cdot \text { all permutations of } m_{1}, m_{2}, m_{3}\right\} \\
& \left.+(-1)^{P} \cdot \text { inequivalent permutations of } \mu, \nu, \rho, \sigma\right] \\
& -\left[\left(C_{\sigma}^{m_{3}} C_{\rho}^{m_{2}} C_{\nu}^{m_{1}} \partial_{\mu} \lambda_{1}+(-1)^{P} \cdot \text { all permutations of } m_{1}, m_{2}, m_{3}\right)\right. \\
& \left.+(-1)^{P} \cdot \text { cyclic permutations of } \mu, \nu, \rho, \sigma\right] .
\end{aligned}
$$

In terms of these field strengths, the second term of the action (4.1) may be written 


$$
\begin{aligned}
S_{2} \equiv & \int \sqrt{-\operatorname{det} G_{F}^{(10)}} e^{\Phi^{(10)} / 3}\left(-\frac{1}{2 \cdot 7 !}\right) G_{F}^{(10) M_{1} N_{1}} \ldots G_{F}^{(10) M_{7} N_{7}} K_{M_{1} \ldots M_{7}}^{(10)} K_{N_{1} \ldots N_{7}}^{(10)} \\
= & -\int \sqrt{-g}\left[\frac{1}{2\left(\lambda_{2}\right)^{2}} g^{\mu \nu} \partial_{\mu} \lambda_{1} \partial_{\nu} \lambda_{1}\right. \\
& +\frac{1}{4 \lambda_{2}} \hat{G}_{m_{1} m_{2}} g^{\mu \rho} g^{\nu \sigma}\left(F_{\mu \nu}^{(\mathcal{D}) m_{1}}+\lambda_{1} F_{\mu \nu}^{(C) m_{1}}\right)\left(F_{\rho \sigma}^{(\mathcal{D}) m_{2}}+\lambda_{1} F_{\rho \sigma}^{(C) m_{2}}\right) \\
& +\frac{1}{2 \cdot 2 ! \cdot 3 !} \hat{G}_{m_{1} n_{1}} \hat{G}_{m_{2} n_{2}} g^{\mu_{1} \nu_{1}} \ldots g^{\mu_{3} \nu_{3}} \hat{K}_{\mu_{1} \mu_{2} \mu_{3}}^{m_{1} m_{2}} \hat{K}_{\nu_{1} \nu_{2} \nu_{3}}^{n_{1} n_{2}} \\
& \left.+\frac{\lambda_{2}}{2 \cdot 3 ! \cdot 4 !} \hat{G}_{m_{1} n_{1}} \ldots \hat{G}_{m_{3} n_{3}} g^{\mu_{1} \nu_{1}} \ldots g^{\mu_{4} \nu_{4}} \mathcal{K}_{\mu_{1} \ldots \mu_{4}}^{m_{1} \ldots m_{3}} \mathcal{K}_{\nu_{1} \ldots \nu_{4}}^{n_{1} \ldots n_{3}}\right] .
\end{aligned}
$$

We would like to compare the sum of the actions given in eqs.(4.6) and (A.5) with the action given in eq.(3.30). In order to do this, we need to dualize the threeand four-form field strengths $\hat{K}_{\mu_{1} \mu_{2} \mu_{3}}^{m_{1} m_{2}}$ and $\mathcal{K}_{\mu_{1} \ldots \mu_{4}}^{m_{1} \ldots m_{3}}$. We start with the four-form field strength. The equation of motion of the field $\mathcal{C}_{\nu_{2} \nu_{3} \nu_{4}}^{m_{1} m_{2} m_{3}}$ is given by

$$
\partial_{\nu_{1}}\left[\lambda_{2} \sqrt{-g} \hat{G}_{m_{1} n_{1}} \ldots \hat{G}_{m_{3} n_{3}} g^{\mu_{1} \nu_{1}} \ldots g^{\mu_{4} \nu_{4}} \mathcal{K}_{\mu_{1} \ldots \mu_{4}}^{m_{1} \ldots m_{3}}\right]=0
$$

Since $\mathcal{K}_{\mu_{1} \ldots \mu_{4}}^{m_{1} \ldots m_{3}}$ is antisymmetric in $\mu_{1}, \ldots \mu_{4}$, we may write

$$
\lambda_{2} \sqrt{-g} \hat{G}_{m_{1} n_{1}} \ldots \hat{G}_{m_{3} n_{3}} g^{\mu_{1} \nu_{1}} \ldots g^{\mu_{4} \nu_{4}} \mathcal{K}_{\mu_{1} \ldots \mu_{4}}^{m_{1} \ldots m_{3}}=\epsilon^{\nu_{1} \ldots \nu_{4}} H_{n_{1} n_{2} n_{3}}
$$

for some $H_{n_{1} n_{2} n_{3}}$. Eq.(A.6) then gives,

$$
\partial_{\nu} H_{n_{1} n_{2} n_{3}}=0
$$

showing that $H_{n_{1} n_{2} n_{3}}$ is a constant. Comparison with the original formulation of the theory shows that $H_{m n p}$ denotes the components of the three-form field strength in the internal directions, and hence are quantized [27]. Furthermore, in the original formulation of the theory these constants were set to zero; hence if we 
want to describe the same field configurations, we must set these constants to zero in this theory as well. This gives

$$
\mathcal{K}_{\mu_{1} \ldots \mu_{4}}^{m_{1} m_{2} m_{3}}=0
$$

Let us now turn to the $\mathcal{B}_{\nu_{2} \nu_{3}}^{m_{1} m_{2}}$ equations of motion. ${ }^{\star}$ This is given by

$$
\partial_{\nu_{1}}\left(\sqrt{-g} \hat{G}_{m_{1} n_{1}} \hat{G}_{m_{2} n_{2}} g^{\mu_{1} \nu_{1}} \ldots g^{\mu_{3} \nu_{3}} \hat{K}_{\mu_{1} \mu_{2} \mu_{3}}^{m_{1} m_{2}}\right)=0
$$

This gives

$$
\sqrt{-g} \hat{G}_{m_{1} n_{1}} \hat{G}_{m_{2} n_{2}} g^{\mu_{1} \nu_{1}} \ldots g^{\mu_{3} \nu_{3}} \hat{K}_{\mu_{1} \mu_{2} \mu_{3}}^{m_{1} m_{2}}=\epsilon^{\nu_{1} \nu_{2} \nu_{3} \sigma} \partial_{\sigma} \hat{B}_{n_{1} n_{2}}
$$

for some $\hat{B}_{n_{1} n_{2}}$. The Bianchi identity for the field strength $\hat{K}_{\mu \nu \rho}^{m_{1} m_{2}}$

$$
\epsilon^{\mu \nu \rho \sigma} \partial_{\sigma} \hat{K}_{\mu \nu \rho}^{m_{1} m_{2}}=-\frac{3}{2} \epsilon^{\mu \nu \rho \sigma}\left(F_{\rho \sigma}^{(C) m_{1}} F_{\mu \nu}^{(\mathcal{D}) m_{2}}-F_{\rho \sigma}^{(C) m_{2}} F_{\mu \nu}^{(\mathcal{D}) m_{1}}\right),
$$

as derived from eq.(A.3), gives rise to the following equation of motion for the field $\hat{B}_{m_{1} m_{2}}$

$$
\partial_{\sigma}\left(\sqrt{-g} g^{\sigma \rho} \hat{G}^{m_{1} n_{1}} \hat{G}^{m_{2} n_{2}} \partial_{\rho} \hat{B}_{n_{1} n_{2}}\right)=\frac{1}{4} \epsilon^{\mu \nu \rho \sigma}\left(F_{\rho \sigma}^{(C) m_{1}} F_{\mu \nu}^{(\mathcal{D}) m_{2}}-F_{\rho \sigma}^{(C) m_{2}} F_{\mu \nu}^{(\mathcal{D}) m_{1}}\right) .
$$

It can be checked easily that the contributions to all the equations of motion and Bianchi identities derived from the term involving $\hat{K}_{\mu_{1} \mu_{2} \mu_{3}}^{m_{1} m_{2}}$ in the action (A.5) are

$\star$ Although we are carrying out this dualization in order to compare the dimensionally reduced action to the action $(3.30), \mathrm{SL}(2, \mathrm{R})$ invariance of the dimensionally reduced action can be seen without this dualization, in the same way that in the usual scheme $\mathrm{O}(6,22)$ symmetry of the action can be seen without dualizing the $B_{\mu \nu}$ field. 
identical to the ones derived from the action

$$
\begin{array}{r}
-\int d^{4} x \sqrt{-g}\left[\frac{1}{4} \hat{G}^{m_{1} n_{1}} \hat{G}^{m_{2} n_{2}} g^{\mu \nu} \partial_{\mu} \hat{B}_{m_{1} m_{2}} \partial_{\nu} \hat{B}_{n_{1} n_{2}}\right. \\
\left.+\frac{1}{2} \hat{B}_{m_{1} m_{2}} F_{\mu \nu}^{(C) m_{1}} \tilde{F}_{\rho \sigma}^{(\mathcal{D}) m_{2}} g^{\mu \rho} g^{\nu \sigma}\right] .
\end{array}
$$

Combining eqs.(4.6), (A.5), (A.9) and (A.14) we get the final form of the action:

$$
\begin{aligned}
S=\int d^{4} x \sqrt{-g}[ & R-\frac{1}{2\left(\lambda_{2}\right)^{2}} g^{\mu \nu} \partial_{\mu} \lambda \partial_{\nu} \bar{\lambda} \\
& +\frac{1}{4} g^{\mu \nu} \partial_{\mu} \hat{G}_{m n} \partial_{\nu} \hat{G}^{m n}-\frac{1}{4} g^{\mu \nu} \hat{G}^{m_{1} m_{2}} \hat{G}^{n_{1} n_{2}} \partial_{\mu} \hat{B}_{m_{1} n_{1}} \partial_{\nu} \hat{B}_{m_{2} n_{2}} \\
& -\frac{1}{4 \lambda_{2}} \hat{G}_{m_{1} m_{2}} g^{\mu \rho} g^{\nu \sigma}\left\{|\lambda|^{2} F_{\mu \nu}^{(C) m_{1}} F_{\rho \sigma}^{(C) m_{2}}\right. \\
& \left.+2 \lambda_{1} F_{\mu \nu}^{(C) m_{1}} F_{\rho \sigma}^{(\mathcal{D}) m_{2}}+F_{\mu \nu}^{(\mathcal{D}) m_{1}} F_{\rho \sigma}^{(\mathcal{D}) m_{2}}\right\} \\
& \left.-\frac{1}{2} \hat{B}_{m_{1} m_{2}} F_{\mu \nu}^{(C) m_{1}} \tilde{F}_{\rho \sigma}^{(\mathcal{D}) m_{2}} g^{\mu \rho} g^{\nu \sigma}\right] .
\end{aligned}
$$

This action is identical to to the one given in eq.(3.30), provided that we identify the vector fields $A_{\mu}^{(m, 1)}, A_{\mu}^{(m, 2)}$ appearing in eq.(3.30) with $C_{\mu}^{m}$ and $\mathcal{D}_{\mu}^{m}$, respectively.

\section{REFERENCES}

[1] C. Montonen and D. Olive, Phys. Lett. B72 (1977) 117; P. Goddard, J. Nuyts and D. Olive, Nucl. Phys. B125 (1977) 1.

[2] H. Osborn, Phys. Lett. B83 (1979) 321.

[3] A. Strominger, Nucl. Phys. B343 (1990) 167; C. Callan, J. Harvey and A. Strominger, Nucl. Phys. B359 (1991) 611; B367 (1991) 60; preprint EFI-91-66 (hep-th/9112030).

[4] M. Duff, Class. Quantum Grav. 5 (1988) 189; M. Duff and J. Lu, Nucl. Phys. B354 (1991) 141; Phys. Rev. Lett. 66 (1991) 1402; Class. Quantum Grav. 9 (1991) 1; M. Duff, R. Khuri and J. Lu, Nucl. Phys. B377 (1992) 281; J. Dixon, M. Duff and J. Plefka, Phys. Rev. Lett. 69 (1992) 3009. 
[5] M. Duff and J. Lu, Nucl. Phys. B354 (1991) 129.

[6] M. Duff and J. Lu, Nucl. Phys. B357 (1991) 354.

[7] S. Ferrara, J. Scherk and B. Zumino, Nucl. Phys. B121 (1977) 393; E. Cremmer, J. Scherk and S. Ferrara, Phys. Lett. B68 (1977) 234; E. Cremmer and J. Scherk, Nucl. Phys. B127 (1977) 259; E. Cremmer and B. Julia, Nucl. Phys. B159 (1979) 141; E. Cremmer, J. Scherk and J. Schwarz, Phys. Lett. B84 (1979) 83.

[8] E. Cremmer, J. Scherk and S. Ferrara, Phys. Lett. B74 (1978) 61; M. Gaillard and B. Zumino, Nucl. Phys. B193 (1981) 221; M. De Roo, Nucl. Phys. B255 (1985) 515.

[9] J. Maharana and J. Schwarz, Nucl. Phys. B390 (1993) 3.

[10] K. Narain, Phys. Lett. B169 (1986) 41.

[11] K. Narain, H. Sarmadi and E. Witten, Nucl. Phys. B279 (1987) 369.

[12] A. Shapere, S. Trivedi and F. Wilczek, Mod. Phys. Lett. A6 (1991) 2677.

[13] A. Sen, preprint TIFR-TH-92-41 (hep-th/9207053) (to appear in Nucl. Phys. B).

[14] J. Schwarz, preprint CALT-68-1815 (hep-th/9209125).

[15] A. Font, L. Ibáñez, D. Lust and F. Quevedo, Phys. Lett. B249 (1990) 35; S.J. Rey, Phys. Rev. D43 (1991) 526.

[16] A. Dabholkar, G. Gibbons, J. Harvey and F. Ruiz, Nucl. Phys. B340 (1990) 33; A. Dabholkar and J. Harvey, Phys. Rev. Lett. 63 (1989) 719.

[17] A. Sen, Nucl. Phys. B388 (1992) 457.

[18] A. Sen, Phys. Lett. B303 (1993) 22.

[19] A. Sen, preprint NSF-ITP-93-29 (hep-th/9303057).

[20] M. Henneaux and C. Teitelboim, p. 79 in Proc. Quantum Mechanics of Fundamental Systems 2 (Santiago 1987); Phys. Lett. B206 (1988) 650. 
[21] R. Floreanini and R. Jackiw, Phys. Rev. Lett. 59 (1987) 1873.

[22] A. Tseytlin, Phys. Lett. B242 (1990) 163; Nucl. Phys. B350 (1991) 395.

[23] R. Kallosh and T. Ortin, preprint SU-ITP-93-3 (hep-th/9302109).

[24] N. Marcus and J. Schwarz, Nucl. Phys. B228 (1983) 145.

[25] A. Chamseddine, Phys. Rev. D24 (1981) 3065; S. Gates and H. Nishino, Phys. Lett. B173 (1986) 52; A. Salam and E. Sezgin, Physica Scripta 32 (1985) 283.

[26] J. Gauntlett, J. Harvey and J. Liu, preprint EFI-92-67 (hep-th/9211056).

[27] R. Rohm and E. Witten, Ann. Phys. (NY) 170 (1986) 454. 